Supporting Information for

\title{
Impact of Particle Size on Droplet Coalescence in Solid-Stabilized High Internal Phase Emulsions
}

\author{
Max Kaganyuk and Ali Mohraz*
}

Department of Chemical and Biomolecular Engineering, University of

California, Irvine, California 92697, United States

Email:mohraz@uci.edu 


\section{Table of Contents}

Appendix A. Interface fractions

Figure A1. Experimental emulsion sample

Appendix B. Capillary pressure model

S6

Figure B1. Particle-stabilized films

Appendix C. Film volume

Figure B1. Film unit cells

Figure B2. Monolayer film

Figure B3. Bilayer film

Figure S1. Scanning electron microscopy images of particles $\quad$ S15

Figure S2. Particle contact angle as measured by the immersed droplet method. S15

Figure S3. Experimental water-in-oil emulsion sample. $\quad$ S16

Figure S4. Characteristic HIPE sample. $\quad$ S16 


\section{Appendix A. Interface fractions}
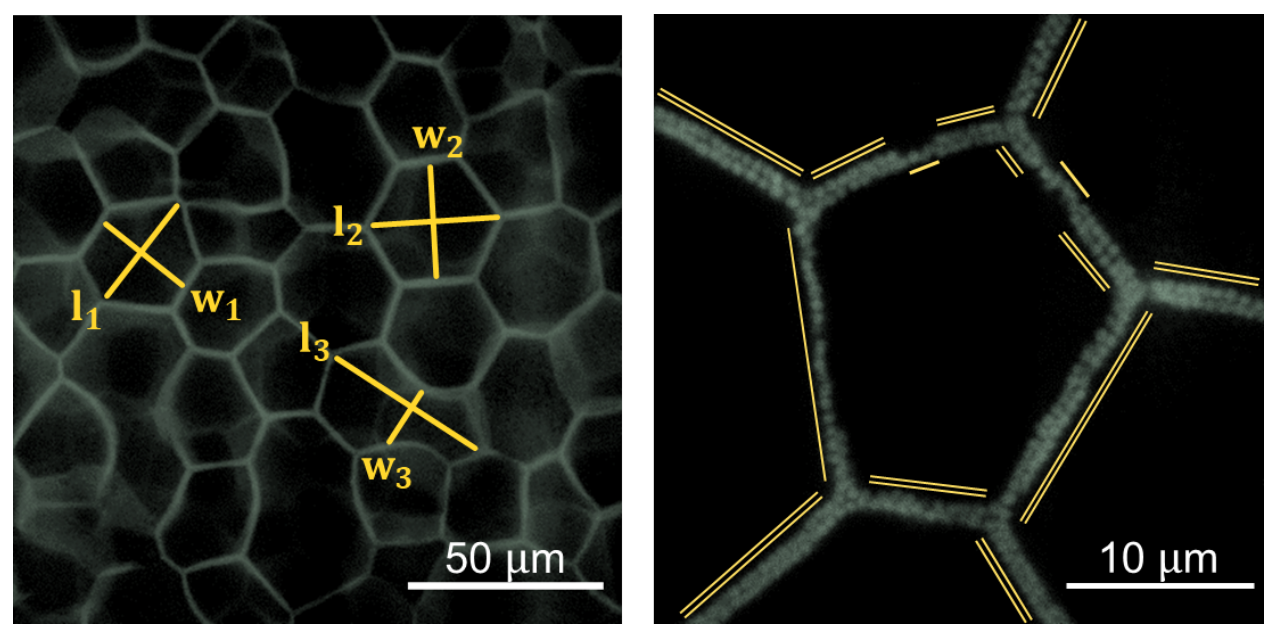

Figure A1: A high internal phase emulsion sample imaged with a 20X objective (left) and a 100X objective (right).

Figure A1 shows confocal images of an experimental sample, taken at different magnifications. As discussed within the main text, and illustrated in Figure A1, droplet aspect ratios can be quantified from the images taken with a $20 \mathrm{X}$ objective. For each droplet, $\mathrm{l}_{\mathrm{i}}$ and $\mathrm{w}_{\mathrm{i}}$ represent its greatest length and width, respectively, while the ratio of the two is its aspect ratio, $\alpha_{\mathrm{i}}$ (Note that $\mathrm{W}_{\mathrm{i}}$, is measured perpendicular to $\mathrm{l}_{\mathrm{i}}$ at its median). This data has been recorded for $\sim 50$ droplets in each experimental sample reported in the main text. For high internal phase emulsion (HIPE) samples, $\phi_{\text {eff }}>0.80$, images were also taken with a $100 \mathrm{X}$ objective, which allow visual observation of the different particle stabilized interfaces present. Here we will show how we can estimate the fraction of interfaces that had ruptured in our HIPE samples, as well as the fraction of interfaces that are stabilized by either a particle monolayer or particle bilayer. From the droplet aspect ratio analysis done in the main text, it was argued that droplets with an aspect ratio greater than 2 were a result of droplet coalescence. The total length of interfaces that constituted unstable films which ruptured is taken as twice the sum of $\mathrm{w}_{\mathrm{i}}$ for all droplets with aspect ratios greater than 2 .

$$
\mathrm{L}_{\mathrm{r}}=\sum_{1}^{\mathrm{N}_{\mathrm{d}}\left(\alpha_{\mathrm{i}}>2\right)} 2 \mathrm{w}_{\mathrm{i}}\left(\alpha_{\mathrm{i}}>2\right)
$$


To determine the total length of stable interfaces in a HIPE sample the perimeters of all the droplets, examined for their aspect ratio, are estimated and summed.

$$
\mathrm{L}_{\mathrm{s}}=\sum_{1}^{\mathrm{N}_{\mathrm{d}}} \rho_{\mathrm{i}}
$$

The perimeter of each droplet, $\rho_{\mathrm{i}}$, is evaluated by taking its $\mathrm{l}_{\mathrm{i}}$ and $\mathrm{w}_{\mathrm{i}}$ as the major and minor axis of an ellipse, respectively, and utilizing the Ramanujan approximation shown below.

$$
\rho_{\mathrm{i}} \approx \pi\left(\mathrm{l}_{\mathrm{i}}+\mathrm{w}_{\mathrm{i}}\right)\left(1+\frac{3 \lambda}{10+\sqrt{4-3 \lambda}}\right)
$$

The parameter $\lambda$ is given by the following.

$$
\lambda=\frac{\left(\mathrm{l}_{\mathrm{i}}-\mathrm{w}_{\mathrm{i}}\right)^{2}}{\left(\mathrm{l}_{\mathrm{i}}+\mathrm{w}_{\mathrm{i}}\right)^{2}}
$$

The fraction of ruptured interfaces, $\mathrm{X}_{\mathrm{r}}$, is written out as seen in the main text (equation 1), by utilizing equations $\mathrm{A} 1$ and $\mathrm{A} 2$.

$$
\mathrm{X}_{\mathrm{r}}=\frac{\sum_{1}^{\mathrm{N}_{\mathrm{d}}\left(\alpha_{\mathrm{i}}>2\right)} 2 \mathrm{w}_{\mathrm{i}}\left(\alpha_{\mathrm{i}}>2\right)}{\sum_{1}^{\mathrm{N}_{\mathrm{d}}} \rho_{\mathrm{i}}+\sum_{1}^{\mathrm{N}_{\mathrm{d}}\left(\alpha_{\mathrm{i}}>2\right)} 2 \mathrm{w}_{\mathrm{i}}\left(\alpha_{\mathrm{i}}>2\right)}
$$

Equation (A5) was utilized in generating the error bars shown in Figure 7 of the main text. For each sample plotted in Figure 7, four different sets of $\sim 25$ droplets were analyzed for their droplet aspect ratio data, used to estimate $\mathrm{X}_{\mathrm{r}}$. The average and standard deviation of these four values are plotted in Figure 7.

In order to calculate the fraction of stable interfaces, $1-\mathrm{X}_{\mathrm{r}}$, that are stabilized by either a particle monolayer or bilayer, sample images taken with the 100X objective are analyzed. As seen in Figure A1, the different films are denoted by single lines for monolayers and double lines for bilayers. 
Their respective lengths, $m_{i}$ and $b_{i}$, are summed up to give the total amount of monolayer and bilayer stabilized interfaces, as shown below.

$$
\begin{aligned}
& \mathrm{L}_{\mathrm{m}}=\sum_{1}^{\mathrm{N}_{\mathrm{m}}} 2 \mathrm{~m}_{\mathrm{i}} \\
& \mathrm{L}_{\mathrm{b}}=\sum_{1}^{\mathrm{N}_{\mathrm{b}}} 2 \mathrm{~b}_{\mathrm{i}}
\end{aligned}
$$

The film lengths in equations A6 and A7 are multiplied by 2 to capture the two droplet interfaces present in each film. For each sample the total amount of $L_{m}$ and $L_{b}$ analyzed is $\sim 1000 \mu \mathrm{m}$. Equations A5-A7 are used to express the fraction of interfaces stabilized by particle monolayers, $\mathrm{X}_{\mathrm{m}}$, as found in the main text (equation 2).

$$
\mathrm{X}_{\mathrm{m}}=\left(1-\mathrm{X}_{\mathrm{r}}\right) \frac{\sum_{1}^{\mathrm{N} m} \mathrm{~m}_{\mathrm{i}}}{\sum_{1}^{\mathrm{N}_{\mathrm{m}}} \mathrm{m}_{\mathrm{i}}+\sum_{1}^{\mathrm{N}_{\mathrm{b}}} \mathrm{b}_{\mathrm{i}}}
$$

For the samples plotted in Figure 7, four different sets of $L_{m}$ and $L_{b}$ were measured, each summing up to $\sim 250 \mu \mathrm{m}$, to evaluate $\mathrm{X}_{\mathrm{m}}$ (The average $\mathrm{X}_{\mathrm{r}}$ for each sample was used in calculating $\mathrm{X}_{\mathrm{m}}$ ). The average and standard deviation of these four $\mathrm{X}_{\mathrm{m}}$ values are plotted in Figure 7.

Note, the fraction of particle bilayer stabilized interfaces, $\mathrm{X}_{\mathrm{b}}$, can be determined from $\mathrm{X}_{\mathrm{r}}$ and $\mathrm{X}_{\mathrm{m}}$.

$$
\mathrm{X}_{\mathrm{b}}=1-\mathrm{X}_{\mathrm{r}}-\mathrm{X}_{\mathrm{m}}
$$




\section{Appendix B. Capillary pressure model}

Our derivation for the capillary pressure that arises during the thinning of a liquid film between particle-coated droplets follows the theoretical model reported by G. Kaptay'. This model simplifies the shape of the menisci infiltrating the pores formed between neighboring interfacial particles, allowing the capillary pressure at different extents of film thinning to be evaluated geometrically. Two configurations are considered for the stabilizing particles present in the liquid film between droplets: particle monolayer and particle bilayer. For both, the meniscus going through a pore created by three spherical particles is approximated as a segment of a sphere (spherical cap). The two particle configurations are illustrated below in Figure B1 (Figure 7 of the main text). Note, the illustrations show a cross-sectional view of the particle-stabilized films, where the two droplets interfaces are at the top and bottom of the particle layers.
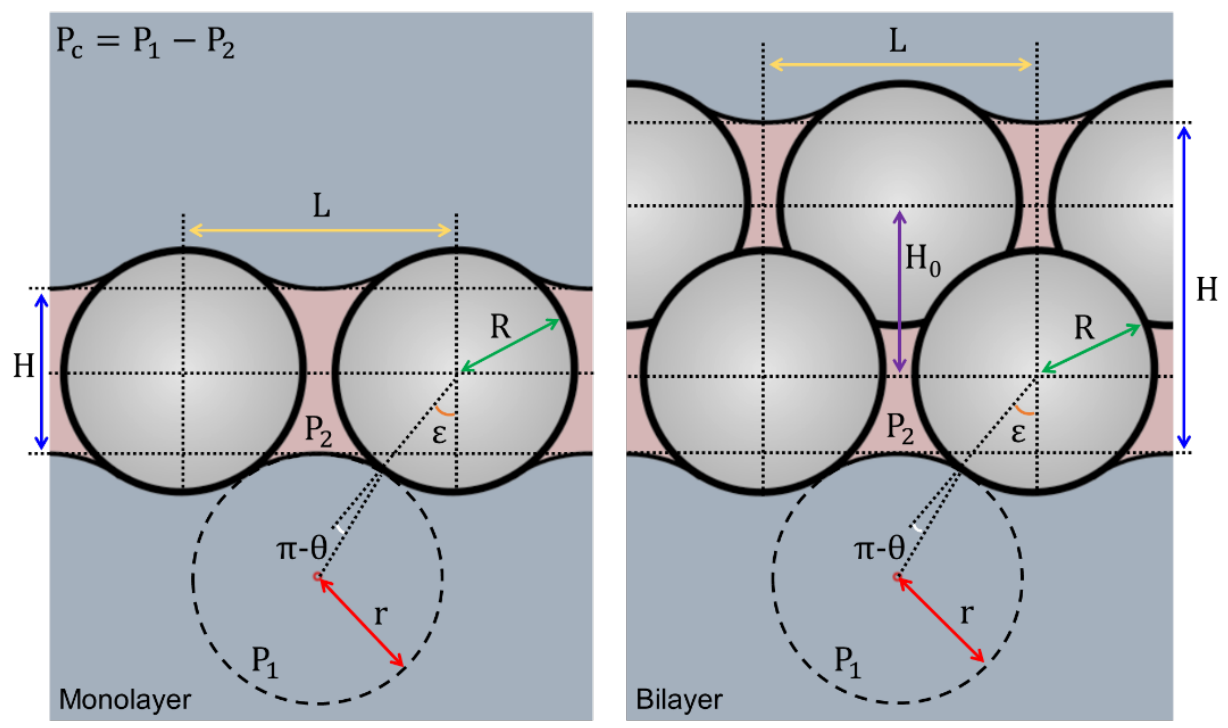

Figure B1: Illustrations of the two configurations in which particles can stabilize the liquid film formed between two droplets.

The known or set parameters in Figure B1 are: separation distance between particle centers, L, particle radius, $R$, infiltration angle, $\varepsilon$, and particle contact angle, $\theta$, as measured against the aqueous fluid phase (the dispersed phase for our experimental system). $\mathrm{P}_{1}$ represents the pressure 
in the droplet phase and $\mathrm{P}_{2}$ is the pressure in the liquid film. Capillary pressure, $\mathrm{P}_{\mathrm{c}}$, is the difference between $\mathrm{P}_{1}$ and $\mathrm{P}_{2}$. At the lowest possible $\varepsilon, \varepsilon_{0}=\pi-\theta$, the capillary pressure is zero and the meniscus is flat. To determine $P_{c}$ at a degree of infiltration above $\varepsilon_{0}$ the assumed spherical shape of the infiltrating meniscus, and Young-Laplace equation are utilized. First, the radius of the sphere that the meniscus is taken to be a segment of, $r$, is determined geometrically. Its equation at a specified $\varepsilon$ is given below.

$$
\mathrm{r}=\left(\frac{\mathrm{L}}{\sqrt{3}}-\mathrm{R} \sin (\varepsilon)\right) / \sin (\varepsilon-(\pi-\theta))
$$

Once $r$ is determined, the Young-Laplace equation is employed to calculate $\mathrm{P}_{c}$ as shown below.

$$
\mathrm{P}_{\mathrm{c}}=\frac{2 \sigma}{\mathrm{r}}
$$

Here $\sigma$ represents the interfacial tension between the fluids in the droplet and film. Scaling $\mathrm{P}_{c}$ by $2 \sigma / \mathrm{R}$ gives the equation for the dimensionless capillary pressure, $\mathrm{P}_{\mathrm{c}}^{\prime}$, as reported in the main text (equation 4).

$$
\mathrm{P}_{\mathrm{c}}^{\prime}=\sin (\varepsilon-(\pi-\theta)) /\left(\frac{\mathrm{L}}{R \sqrt{3}}-\sin (\varepsilon)\right)
$$

It is important to note that with the simplified model used, equation B3 applies for films stabilized by either a particle monolayer or bilayer. Though the same $\mathrm{P}_{c}^{\prime}$ is found at a set $\varepsilon$, for either particle configuration, the film thickness, $\mathrm{H}$, at which this occurs will differ. As with $\mathrm{r}$, an equation for a $\mathrm{H}$ can be geometrically derived for either film type as given below.

$$
H_{m}=2 R \cos (\varepsilon)+3\left(\frac{L}{\sqrt{3}}-R \sin (\pi-\theta)\right)\left(\cot (\varepsilon-(\pi-\theta))-\frac{1}{\sin (\varepsilon-(\pi-\theta))}\right)
$$




$$
\mathrm{H}_{\mathrm{b}}=\mathrm{H}_{\mathrm{m}}+\mathrm{H}_{0}
$$

Equation B4 gives the thickness of a film stabilized by a particle monolayer, $\mathrm{H}_{\mathrm{m}}$, while equation B5 is for a bilayer stabilized film, $\mathrm{H}_{\mathrm{b}}$. In equation $\mathrm{B} 5, \mathrm{H}_{0}$ represents the distance between the medians of each particle layers found in a film stabilized by a bilayer, as seen in Figure B1. $\mathrm{H}_{0}$ depends on the interparticle separation in the two particle layers as found below.

$$
H_{0}=\sqrt{4 R^{2}-\left(\frac{L}{\sqrt{3}}\right)^{2}}
$$

Note at the limit $\mathrm{L}=2 R \sqrt{3}, \mathrm{H}_{0}=0$, meaning the interparticle separation is such that the two particle layers can merge to form a monolayer. Though equations for film thickness have been presented, in our analysis of the difference in stability between monolayer and bilayer stabilized films we do not compare the two film types on this basis. Rather, we argue a volumetric basis is most appropriate for comparing between the two types of films at a consistent degree of film thinning. In the following appendix we outline the equations derived to estimate film volume at different infiltration angles. We may not explicitly consider film thickness, but equations B4 - B6 are utilized within our film volume calculations.

\section{$\underline{\text { References }}$}

[1] G. Kaptay, "On the equation of the maximum capillary pressure induced by solid particles to stabilize emulsions and foams and on the emulsion stability diagrams," Colloids Surf. Physicochem. Eng. Asp., vol. 282-283, pp. 387-401, Jul. 2006. 


\section{Appendix C. Film volume}

In order to adequately compare between films stabilized with either a particle monolayer or bilayer, we examine their thinning behavior on a volumetric basis instead of film thickness. The parameter $V^{\prime}$ represents the volume of the continuous fluid phase (oil phase for our experimental system) present in the film, scaled by the total volume of particles stabilizing the film, $\mathrm{V}_{\mathrm{p}}$. Shown below is the general equation used to evaluate $V^{\prime}$.

$$
\mathrm{V}^{\prime}=\frac{\left(\mathrm{V}_{\mathrm{t}}-\mathrm{V}_{\mathrm{s}}-\mathrm{V}_{\mathrm{a}}\right)}{\mathrm{V}_{\mathrm{p}}}
$$

In this appendix we derive the explicit equations for the parameters seen in equation $\mathrm{C} 1$, which are defined as follows: total volume of a measured film segment, $V_{t}$, the volume of solid particles present in the segment, $\mathrm{V}_{\mathrm{s}}$, and dispersed fluid phase volume present due to the spherical menisci, $\mathrm{V}_{\mathrm{a}}$ (aqueous phase in our experimental system). In outlining the geometric arguments made to derive these volumetric parameters, references to equations in Appendix B are made. In Figure $\mathrm{C} 1$, the unit cells considered for each film type are represented, as viewed from above the particle layer(s).
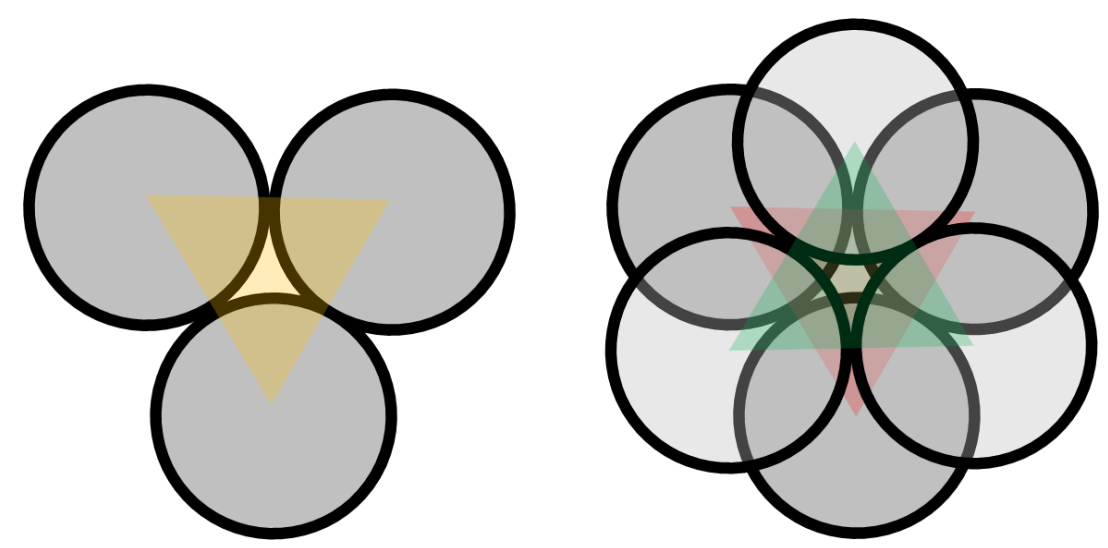

Figure $\mathrm{C} 1$ : The unit cell for a film stabilized by either a particle monolayer (left) or particle bilayer (right) is denoted by the shaded triangles, as viewed from above the particle layer(s). 
The yellow shaded triangle, in Figure C1, outlines the unit cell for a particle monolayer film. The same cell stacked upon itself to create an octahedral hole/pore between the two particle layers, gives the unit cell for a film stabilized by a bilayer, as denoted by the red and green shaded triangles. From these unit cells the total volume of stabilizing particles for a monolayer, $\mathrm{V}_{\mathrm{pm}}$, and bilayer film, $\mathrm{V}_{\mathrm{pb}}$, are determined, noting the spherical particle segments contained within them.

$$
\mathrm{V}_{\mathrm{pb}}=2 \mathrm{~V}_{\mathrm{pm}}=\frac{4 \pi}{3} R^{3}
$$

We will first consider the derivation of equation $\mathrm{C} 1$ for a film stabilized by a particle monolayer, as illustrated in Figure $\mathrm{C} 2$ below.

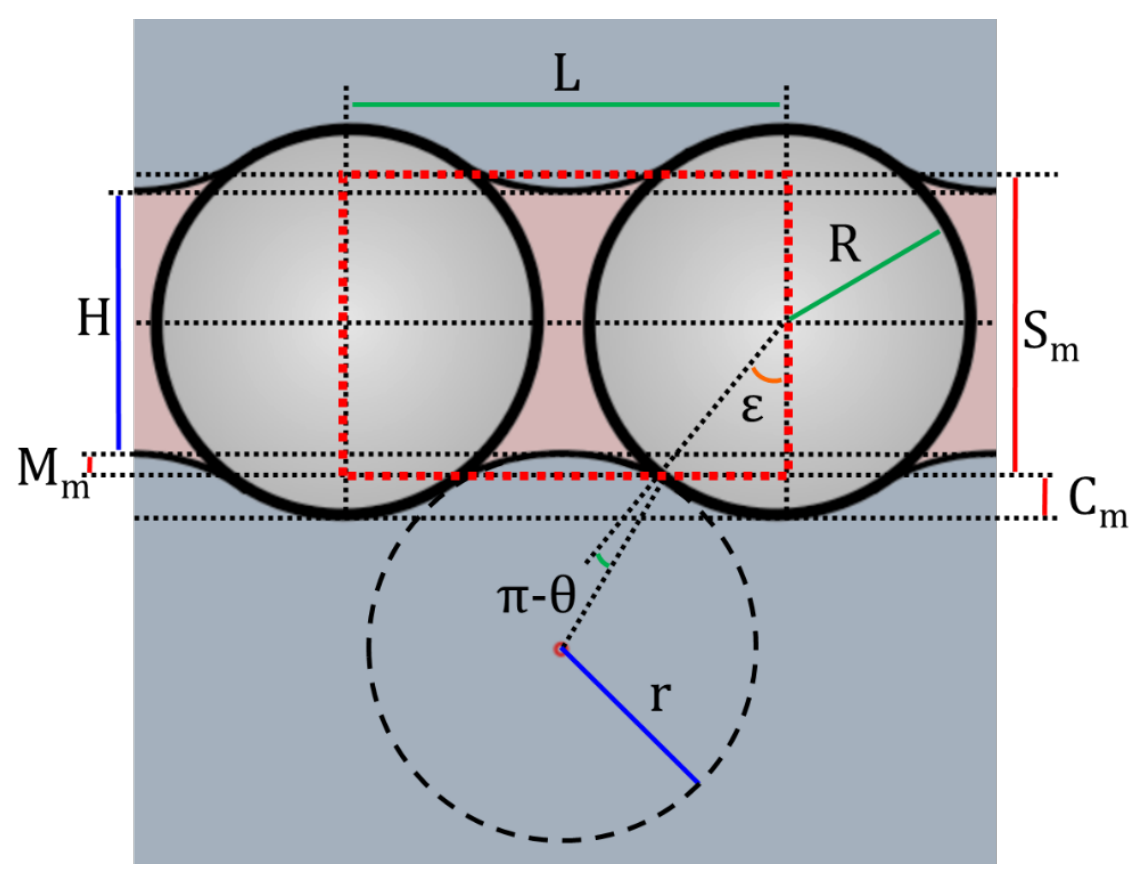

Figure C2: An illustration of film stabilized by a particle monolayer.

In the Figure $\mathrm{C} 2$, the known or set parameters are: separation distance between particle centers, $\mathrm{L}$, particle radius, $R$, infiltration angle, $\varepsilon$, and particle contact angle, $\theta$, as measured against the aqueous fluid phase (the droplet phase for our experimental system). 
The film segment considered for equation $\mathrm{C} 1$ is outlined by the red dashed lines. We can determine the total segment volume for a monolayer stabilized film, $V_{t m}$, by first evaluating its height, $S_{m}$, geometrically from Figure $\mathrm{C} 2$.

$$
\mathrm{S}_{\mathrm{m}}=2 \mathrm{R} \cos (\varepsilon)
$$

$\mathrm{V}_{\mathrm{tm}}$ can then be calculated, recalling that the segment will have a triangular base as seen in Figure C1, with side lengths set by $\mathrm{L}$.

$$
\mathrm{V}_{\mathrm{tm}}=\frac{\sqrt{3}}{4}(L)^{2}\left(\mathrm{~S}_{\mathrm{m}}\right)
$$

The volume in the film segment that is due to the presence of the stabilizing particles, $\mathrm{V}_{\mathrm{sm}}$, is also determined geometrically. From Figure C1, we note that the spherical particles are 1/6 segments within the unit cell. The height of the particle's spherical caps, $C_{m}$, not included in the film segment is governed by $\varepsilon$, as shown below.

$$
\mathrm{C}_{\mathrm{m}}=\mathrm{R}-\mathrm{R} \cos (\varepsilon)
$$

Since the volume of a sphere and spherical cap is known, we can derive an equation for $\mathrm{V}_{\mathrm{sm}}$, the particle volume that is included in our evaluation of $\mathrm{V}_{\mathrm{tm}}$.

$$
\mathrm{V}_{\mathrm{sm}}=\frac{2 \pi}{3} \mathrm{R}^{3}-\frac{\pi}{6}\left(\mathrm{C}_{\mathrm{m}}\right)\left(\left(3(\mathrm{R} \sin (\varepsilon))^{2}\right)+\left(\left(\mathrm{C}_{\mathrm{m}}\right)^{2}\right)\right)
$$

Note in Figure C2, due to the curvature of the infiltrating meniscus some dispersed fluid is included in the total segment volume. To calculate the volume present due to the dispersed fluid phase, $\mathrm{V}_{\mathrm{am}}$, the radius of the spherical meniscus, $r$, and film thickness, $\mathrm{H}$, are first determined (recall equations B1 and B4). The height of the meniscus cap, $\mathrm{M}_{\mathrm{m}}$, included in the film segment can be expressed once $\mathrm{H}$ is known. 


$$
\mathrm{M}_{\mathrm{m}}=\mathrm{R} \cos (\varepsilon)-\frac{\mathrm{H}}{2}
$$

As with $V_{s m}$, the equations for the volume of a sphere and spherical cap are used to derive $V_{a m}$.

$$
\mathrm{V}_{\mathrm{am}}=\frac{2 \pi}{3}\left(\mathrm{M}_{\mathrm{m}}\right)^{2}\left(3 \mathrm{r}-\mathrm{M}_{\mathrm{m}}\right)
$$

With equations for $\mathrm{V}_{\mathrm{pm}}, \mathrm{V}_{\mathrm{tm}}, \mathrm{V}_{\mathrm{sm}}$, and $\mathrm{V}_{\mathrm{am}}$ found, equation $\mathrm{C} 1$ can be rewritten for a film stabilized by a particle monolayer.

$$
V_{m}^{\prime}=\frac{\left(\left[\frac{\sqrt{3}}{4}(L)^{2}\left(S_{m}\right)\right]-\left[\frac{2 \pi}{3} R^{3}-\frac{\pi}{6}\left(C_{m}\right)\left(\left(3(R \sin (\varepsilon))^{2}\right)+\left(\left(C_{m}\right)^{2}\right)\right)\right]-\left[\frac{2 \pi}{3}\left(M_{m}\right)^{2}\left(3 r-M_{m}\right)\right]\right)}{(2 \pi / 3) R^{3}}
$$

The same procedure is followed to derive an equation similar to equation C9 for a film stabilized by a particle bilayer, accounting for the additional film thickness due to $\mathrm{H}_{0}$, as seen in Figure $\mathrm{C}$.

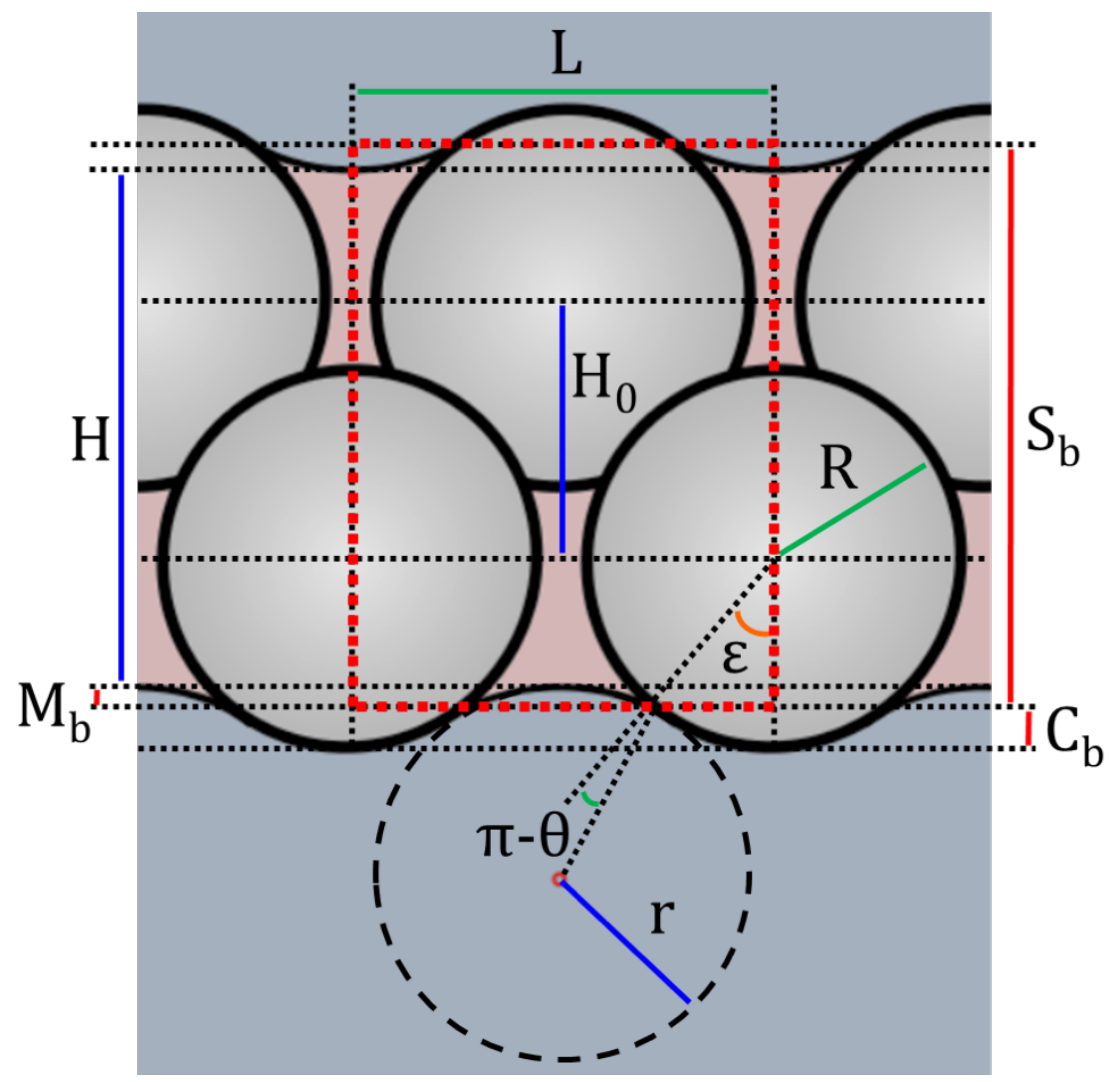

Figure C3: An illustration of film stabilized by a particle bilayer. 
In the Figure C3, the known or set parameters are: separation distance between particle centers, $\mathrm{L}$, particle radius, $\mathrm{R}$, infiltration angle, $\varepsilon$, and particle contact angle, $\theta$, as measured against the aqueous fluid phase (the droplet phase for our experimental system). The height of the film segment, $\mathrm{S}_{\mathrm{b}}$, outlined by the dotted red lines in Figure $\mathrm{C} 3$, is given below. Recall that $\mathrm{H}_{0}$ depends on $\mathrm{L}$ as was shown in equation B6.

$$
\mathrm{S}_{\mathrm{b}}=2 \mathrm{R} \cos (\varepsilon)+\mathrm{H}_{0}
$$

The total film segment volume, $\mathrm{V}_{\mathrm{tb}}$, for a bilayer film is calculated as follows.

$$
\mathrm{V}_{\mathrm{tb}}=\frac{\sqrt{3}}{4}(\mathrm{~L})^{2}\left(\mathrm{~S}_{\mathrm{b}}\right)
$$

The volume included in $\mathrm{V}_{\mathrm{tb}}$, due to the presence of the stabilizing particles, $\mathrm{V}_{\mathrm{sb}}$, is computed considering the volume of spheres and spherical caps, as was done with a monolayer film. Equations for $\mathrm{C}_{\mathrm{b}}$, the height of the particle spherical caps not included in the film segment, and $\mathrm{V}_{\mathrm{sb}}$ are shown below.

$$
\begin{gathered}
\mathrm{C}_{\mathrm{b}}=\mathrm{R}-\mathrm{R} \cos (\varepsilon) \\
\mathrm{V}_{\mathrm{tb}}=\frac{4 \pi}{3} \mathrm{R}^{3}-\frac{\pi}{6}\left(\mathrm{C}_{\mathrm{b}}\right)\left(\left(3(\mathrm{R} \sin (\varepsilon))^{2}\right)+\left(\left(\mathrm{C}_{\mathrm{b}}\right)^{2}\right)\right)
\end{gathered}
$$

$\mathrm{V}_{\mathrm{ab}}$, the dispersed fluid phase present in the total segment volume is also determined considering the volume of spheres and spherical caps. As was done with the monolayer film, the radius of the spherical meniscus, r, and film thickness, H, are first calculated (recall equations B1 and B5). These are used to derive expressions for the height of the meniscus cap, $M_{b}$, included in the film segment and $\mathrm{V}_{\mathrm{ab}}$, as seen below. 


$$
\begin{array}{r}
M_{b}=R \cos (\varepsilon)+\frac{H_{0}-H}{2} \\
V_{a b}=\frac{2 \pi}{3}\left(M_{b}\right)^{2}\left(3 r-M_{b}\right)
\end{array}
$$

With the equations for $\mathrm{V}_{\mathrm{pb}}, \mathrm{V}_{\mathrm{tb}}, \mathrm{V}_{\mathrm{sb}}$, and $\mathrm{V}_{\mathrm{ab}}$ derived, equation $\mathrm{C} 1$ can be rewritten for a film stabilized by a particle bilayer.

$$
V_{b}^{\prime}=\frac{\left(\left[\frac{\sqrt{3}}{4}(L)^{2}\left(S_{b}\right)\right]-\left[\frac{4 \pi}{3} R^{3}-\frac{\pi}{6}\left(C_{b}\right)\left(\left(3(R \sin (\varepsilon))^{2}\right)+\left(\left(C_{b}\right)^{2}\right)\right)\right]-\left[\frac{2 \pi}{3}\left(M_{b}\right)^{2}\left(3 r-M_{b}\right)\right]\right)}{(4 \pi / 3) R^{3}}
$$

Equations $\mathrm{C} 9$ and $\mathrm{C} 16$ are used with equation B3 to generate the nondimensional capillary pressure plots in Figure 9 of the main text. 

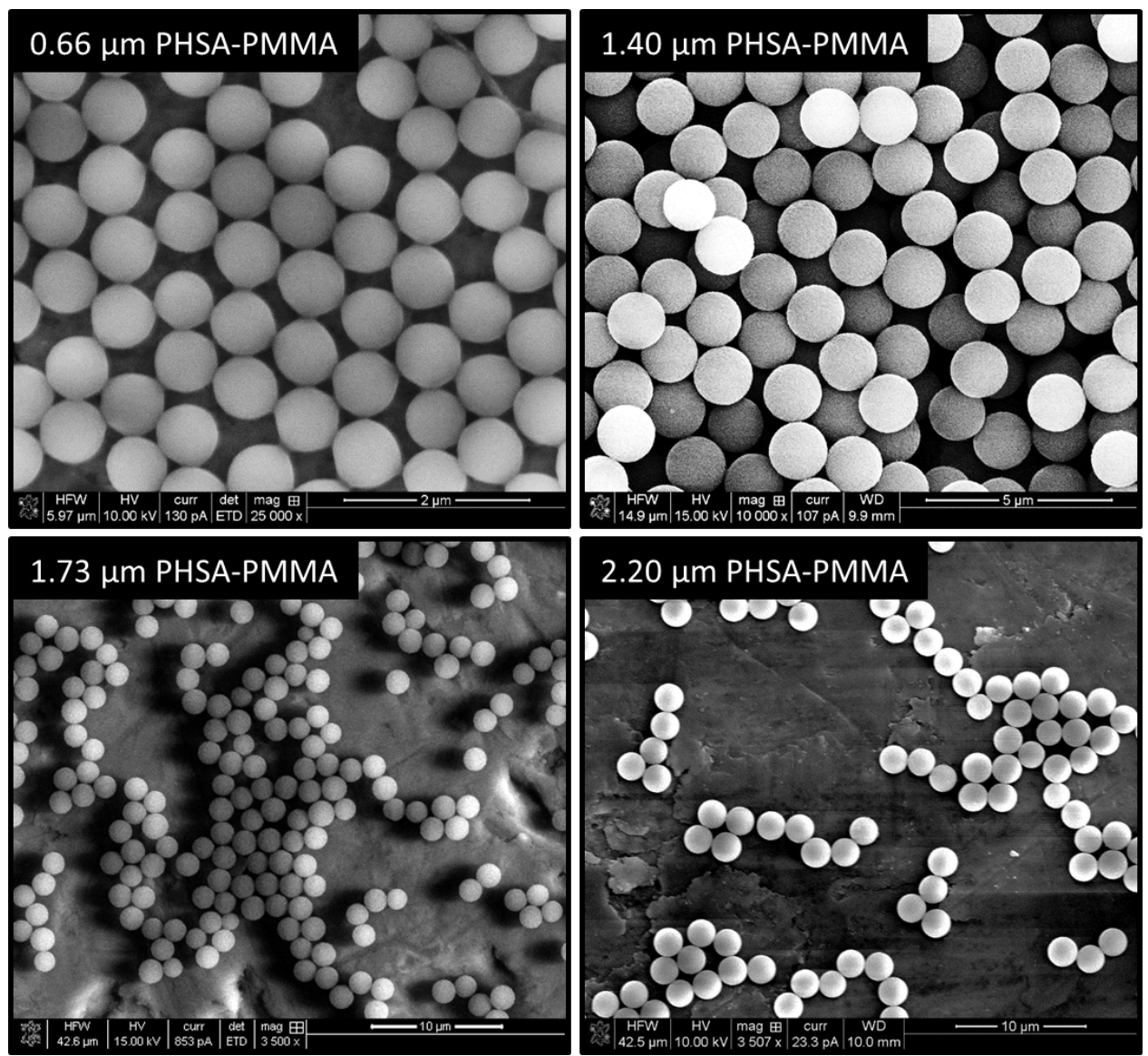

Figure S1. Scanning electron microscopy images of each particle batch utilized in the study. Particles were initially suspended in and cast out of dodecane.
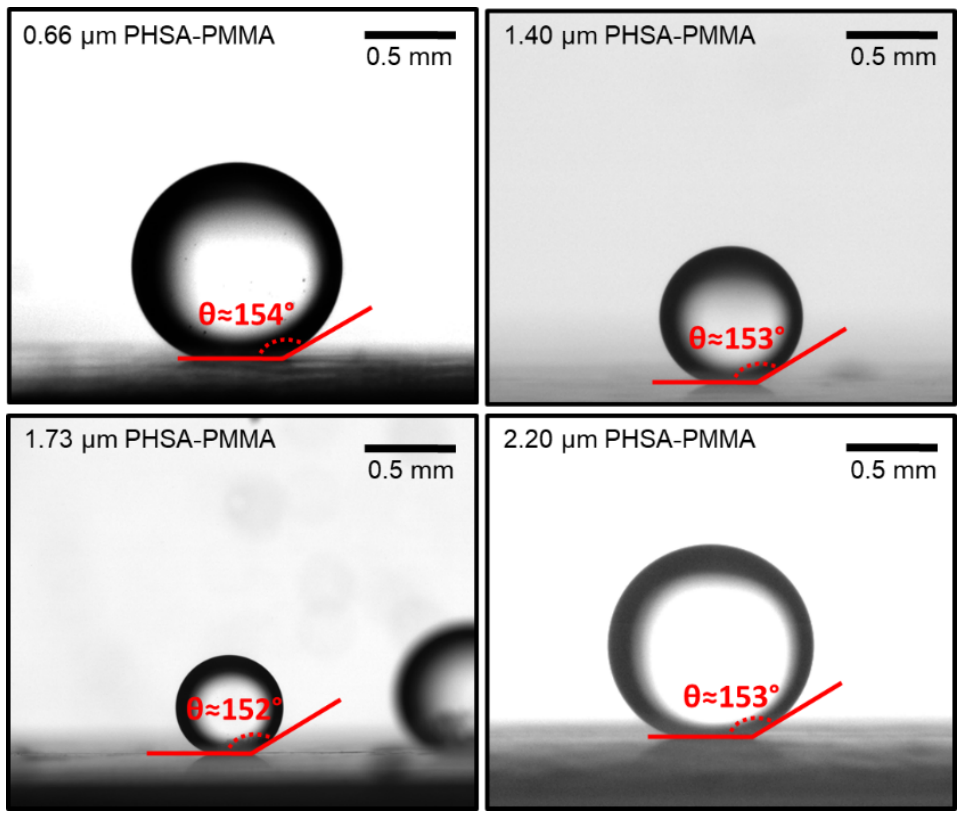

Figure S2. The average static contact angle measured by the immersed droplet method for each batch of particles used in the study. In this method an aqueous droplet sits on a glass coverslip, coated with particles by drop casting, within the organic fluid phase. 


\section{Dodecane}

\section{$10 \mu \mathrm{m}$}

Figure S3. The formation of water-in-oil emulsions is confirmed by selectively dying the aqueous phase with rhodamine B fluorescent dye, which allows it to be distinguished from dodecane via confocal imaging.

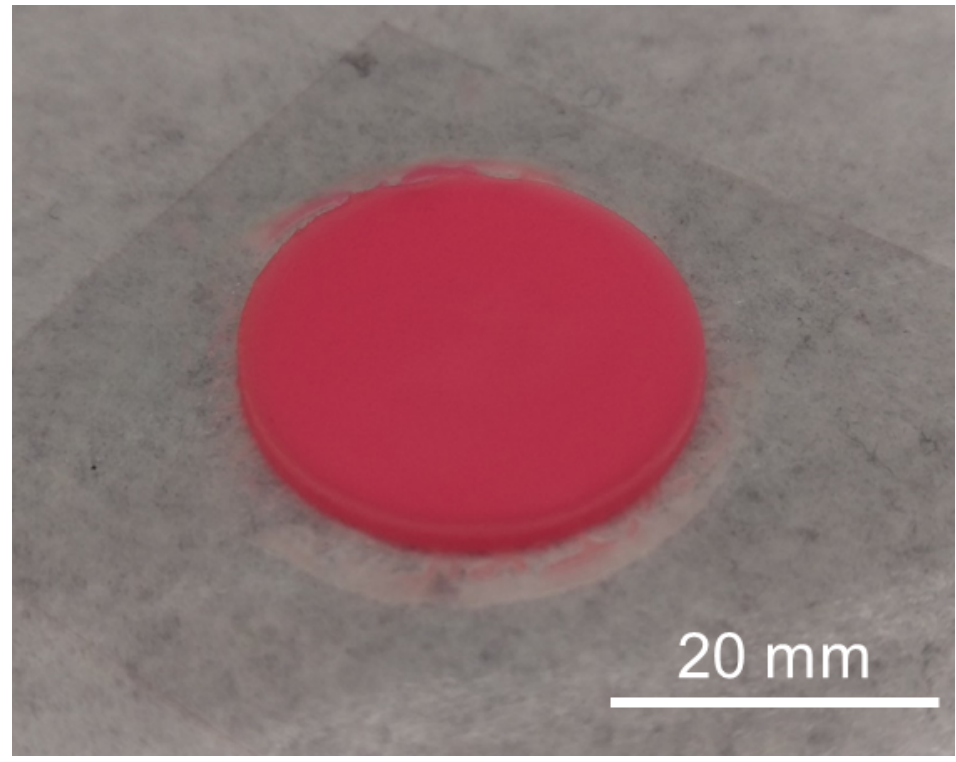

Figure S4. A characteristic emulsion sample, stabilized by PHSA-grafted PMMA particles. The sample shown has been aged for 5 hours to allow the evaporation of hexane, and the cylindrical tube it was initially surrounded by has been removed. The disk-shaped sample has a diameter of $25 \mathrm{~mm}$ and height $\sim 1 \mathrm{~mm}$. 